\title{
ACTIVIDAD ANTIOXIDANTE in vitro DE UN EXTRACTO ACUOSO DE Allium sativum variedad HUARALINO
}

\author{
Silvia Suárez Cunza*, Américo Castro Luna, Neptalí Ale Borja
}

\begin{abstract}
RESUMEN
El objetivo del presente estudio fue evaluar la actividad antioxidante in vitro del bulbo de Allium sativum (ajo) variedad huaralino. Se realizó ensayos para estudiar los metabolitos secundarios azufrados, cuantificando alicina y compuestos orgánicos de bajo peso molecular con grupo tiol. Para los metabolitos oxigenados se evaluó polifenoles y flavonoides. Estos ensayos se realizaron por espectrofotometría. También se cuantificó los minerales por absorción atómica. Los ensayos para estudiar la capacidad antioxidante in vitro se hicieron por formación de carbonilos en albúmina tratada con peróxido de hidrógeno y niveles de especies reactivas al ácido tiobarbitúrico (TBARS) en glóbulos rojos tratados también con peróxido de hidrógeno. Los principales resultados sobre metabolitos azufrados y oxigenados fueron: a) alicina $2,8 \mathrm{mg} / \mathrm{g}$ de bulbo fresco pelado, b) tioles de bajo peso molecular $0,95 \mu \mathrm{mol}$ cisteína (cis)/ g masa seca, c) polifenoles 2,52 mg equivalente ácido gálico/g masa seca y d) $1,71 \mathrm{mg}$ equivalente quercetina/g masa seca. El extracto ejerce protección antioxidante disminuyendo significativamente $(\mathrm{p}<0,05)$ la formación de carbonilos y los niveles de TBARS. En conclusión, el extracto acuoso del bulbo de Allium sativum variedad huaralino tiene metabolitos antioxidantes azufrados y oxigenados con capacidad de ejercer efecto antioxidante in vitro.
\end{abstract}

Palabras clave: Allium sativum, ajo, actividad antioxidante, carbonilo proteico, lipoperoxidación.

\section{ANTIOXIDANT ACTIVITY in vitro OF AQUEOUS EXTRACT OF Allium sativum variety HUARALINO}

\begin{abstract}
The aim of this studio was to evaluate in vitro the bulb antioxidant activity of Allium sativum var. huaralino. Tests were conducted to study the sulfur secondary metabolites, allicin and quantifying organic compounds of low molecular weight with thiol group. For oxygenated metabolites it was evaluated polyphenols and flavonoids. These assays were performed by spectrophotometry. Mineral was also quantified by atomic absorption. Assays for in vitro antioxidant activity were made By carbonyl formation in albumin treated with hydrogen peroxide and thiobarbituric acid reactive species (TBARS) levels in red blood cells also treated with hydrogen peroxide. The main results of sulfur and oxygenated metabolites were: a) allicin $2.8 \mathrm{mg} / \mathrm{g}$ of fresh peeled bulb, b) low molecular weight thiols $\mu \mathrm{mol} 0.95$ cysteine
\end{abstract}

\footnotetext{
C.I. de Bioquímica y Nutrición-Facultad de Medicina-UNMSM

2 Facultad de Farmacia y Bioquímica-UNMSM

3 Facultad de Química e Ingeniería Química-UNMSM

ssuarezc@unmsm.edu.pe
} 
(Cys)/g dry mass, c) $2.52 \mathrm{mg}$ polyphenols EAG / g dry mass d) EQ $1.71 \mathrm{mg} / \mathrm{g}$ dry mass. The extract had antioxidant protection: decreased significantly the formation of protein carbonyl and TBARS levels $(\mathrm{p}<0.05)$. In conclusion, the aqueous extract of Allium sativum var. huaralino bulb has oxygenated and sulfur antioxidant metabolites capable of exerting an antioxidant effect in vitro.

Keywords: Allium sativum, garlic, antioxidant activity, protein carbonyl, lipoperoxidation.

\section{INTRODUCCIÓN}

El hombre, a través de la historia, ha empleado una serie de fuentes naturales, no sólo para su alimentación sino también para paliar una serie de dolencias. Parte de esta riqueza de productos naturales empleados ancestralmente es el Allium sativum (ajo). Éste constituye uno de los recursos vegetales más antiguo del mundo, usado en el arte culinario y como recurso medicinal por sus diversas actividades biológicas y farmacológicas, que incluyen funciones antimicrobiana, antitrombótica, antiateroesclerótica, anticarcinógeno, antiinflamatorio y antioxidante ${ }^{1,2}$.

Allium sativum es una hortaliza cuyo bulbo, de sabor fuerte, especialmente en crudo y ligeramente picante, crece hasta $70 \mathrm{~cm}$ de altura. La raíz bulbosa, está compuesta de 6 a 20 bulbillos, conocidos como "dientes de ajo", reunidos en su base por medio de una película delgada, formando lo que se conoce como "cabeza de ajos". Cada bulbillo se encuentra envuelto por una túnica blanca, a veces algo rojizo, membranoso, transparente y muy delgado, semejante a las que cubren todo el bulbo ${ }^{3}$. Sus metabolitos secundarios más conocidos son compuestos azufrados; también contiene otros tipos de metabolitos como los oxigenados.

Durante el almacenamiento de los bulbos a bajas temperaturas, la aliina se acumula naturalmente. En promedio el bulbo contiene hasta $0,9 \%$ de $\gamma$-glutamilcisteína y hasta 1,8\% aliina. Además de estos principales compuestos azufrados, los bulbos de ajos intactos contienen una pequeña cantidad de S-alil-cisteína (SAC) pero no de alicina.

El SAC es formado del catabolismo de $\gamma$-glutamil cisteína y se le ha reportado como el contribuyente de los beneficios saludables de sus preparaciones. Cuando el ajo es procesado, sea cortándolo o triturándolo, o cuando es deshidratado, pulverizado y luego expuesto al agua, la enzima vacuolar aliinasa (E.C. 4.4.1.4, S-alquil-L-cisteína liasa), rápidamente actúa sobre la aliina, y forma alicina, de olor intenso.

En las últimas décadas se viene estudiando la capacidad antioxidante de diversos productos naturales debido a que los procesos biológicos implican reacciones rédox, especialmente en los organismos aerobios; procesos fisiológicos como el envejecimiento, fisiopatológicos como la respuesta inflamatoria y patológicos como la diabetes o enfermedades cardiovasculares son estados en los que la generación de radicales libres y la defensa antioxidante son claves para conservar la homeostasis de los sistemas biológicos y el balance rédox ${ }^{4}$; estos estudios incluyen trabajos previos en extractos de ajo de diversas variedades ${ }^{5,6}$.

Desde hace relativamente poco tiempo se realiza investigaciones rigurosas para crear la base científica que permita una visión y una utilización que conduzca a darle solidez al uso tradicional y a mejorar las diversas formas de conocimiento popular. La creciente demanda de productos naturales de países como el nuestro en mercados internacionales va en paralelo a exigencias cada vez mayores en cuanto a la calidad del producto en los aspectos físicoquímicos, microbiológicos, genéticos y también en los aspectos bioquímicos, puesto que la optimización de las condiciones de cultivo para conseguir la homogeneidad del recurso tiene que mantener las cualidades terapéuticas por las que se les conoce tradicionalmente. 
El interés de la contribución es aportar conocimientos generados en nuestro país, sobre las propiedades químicas y bioquímicas relacionados con sus capacidades antioxidantes de Allium sativum cultivado en nuestro territorio.

En el presente trabajo se analizó la variedad huaralino del Allium sativum producido por la Estación Experimental Donoso en la provincia de Huaral, departamento de Lima, ubicado a $188 \mathrm{msnm}$ y a $87,6 \mathrm{~km}$ al norte de Lima.

El objetivo principal ha sido la evaluación de la actividad antioxidante in vitro del extracto acuoso de este bulbo mediante técnicas químicas y bioquímicas in vitro.

\section{Muestra}

\section{PARTE EXPERIMENTAL}

Los bulbos de Allium sativum variedad huaralino fueron donados por la Estación Experimental Donoso de la ciudad de Huaral, en los meses de octubre y noviembre del año 2006 y 2007 . Los bulbos tenían en promedio un peso de 51,1 $\pm 12 \mathrm{~g}$, diámetro de 5,72 cm y $14 \pm 3$ bulbillos o dientes por bulbo; el peso promedio por diente fue de 3,64 g. Los reactivos usados en el presente estudio fueron de la casa Sigma-Aldrich Co y de la casa Merck.

Los ensayos mediante espectrofotometría se realizaron en el Centro de Investigación de Bioquímica y Nutrición de la Facultad de Medicina, y las medición de metales por absorción atómica se realizó en la Facultad de Química e Ingeniería Química, ambas de la UNMSM.

\section{Evaluación de metabolitos secundarios azufrados y oxigenados}

Cuantificación de alicina y tioles de bajo peso molecular: Para la cuantificación de la alicina se empleó el método de Mirón 7 usando 4 - mercaptopiridina (4-MP); basado en la formación de un enlace disulfuro mixto, 4-alilmercaptotiopiridina. El ensayo se mide por disminución de la absorbancia del 4-MP a $324 \mathrm{~nm}$ como producto de la reacción con la alicina. El $\varepsilon_{324 \mathrm{~nm}}$ a es $39600 \mathrm{M}^{-1} \mathrm{~cm}^{-1}$. El resultado se obtuvo aplicando la siguiente fórmula ${ }^{7}$ :

[alicina $] \mathrm{mg} / \mathrm{mL}=\left[\mathrm{A}_{324 \mathrm{~nm}}\left(4-\mathrm{MP} \sin\right.\right.$ ajo) $-\mathrm{A}_{324 \mathrm{~nm}}(4-\mathrm{MP}$ con ajo $\left.)\right] \times$ x 0,499

Para este ensayo, la muestra se preparó con bulbos frescos de ajo pelados obteniendo un extracto acuoso con agua bidestilada de $46,67 \% \mathrm{w} / \mathrm{v}$.

Para el análisis de tioles de bajo peso molecular, se procedió de acuerdo a Di Simplicio et al ${ }^{8}$, con algunas modificaciones. El fundamento es la reacción del grupo tiol con el reactivo de ácido dinitrobenzoico (DTNB). Se preparó un extracto de ajo a una concentración de 40 $\mathrm{mg} / \mathrm{mL}$ en etanol absoluto (para precipitar las proteínas) y en presencia de $5 \mathrm{mg}$ de ácido ascórbico (agente reductor).

El reactivo DTNB se preparó a una concentración de $5 \mathrm{mM}$. Se empleó como estándar cisteína, preparando una curva de calibración de cinco concentraciones entre 0,1 a 1,0 mM. En el tubo de reacción se tuvo buffer fosfato de sodio $0,1 \mathrm{M} \mathrm{pH} 7,0$, EDTA $1 \mathrm{mM}$, DTNB 0,5 mM y $100 \mu \mathrm{L}$ de muestra de ajo o estándar. Después de cinco minutos a temperatura ambiente se leyó a 412 $\mathrm{nm}$.

Cuantificación de polifenoles y flavonoides (metabolitos oxigenados): La cuantificación del contenido de polifenoles se realizó siguiendo el procedimiento de la International Organization for Standardization (ISO) 14502-1; primero se procedió a la extracción de estos metabolitos ${ }^{9}$. Se preparó un extracto con ajo en polvo a una concentración de $40 \mathrm{mg} / \mathrm{mL}$ en agua bidestilada a $70^{\circ} \mathrm{C}$, se agitó por 10 minutos. Se centrifugó a 200 g x 10 minutos. Se realizó una segunda extracción con el sobrenadante. Ambos extractos fueron unidos y se ajustó el volumen a $10 \mathrm{~mL}$. Este extracto se diluyó 100 veces con agua bidestilada. Cada tubo de reacción se preparó con $100 \mu \mathrm{L}$ de extracto o referencia y se agrega $500 \mu \mathrm{L}$ de reactivo Folin-Ciocalteu y $400 \mu \mathrm{L}$ de $\mathrm{Na}_{2} \mathrm{CO}_{3} 7,5 \% \mathrm{w} / \mathrm{v}$. La lectura se realizó en espectrofotómetro a $765 \mathrm{~nm}$ contra agua. 
El resultado es expresado como equivalentes de ácido gálico (EAG) en mg/g de materia seca. La concentración de polifenoles en las muestras fue derivada de una curva de ácido gálico de 10 a $50 \mu \mathrm{g} / \mathrm{mL}$. $\left(\mathrm{y}=0,0123 \mathrm{x} ; \mathrm{R}^{2}=0,9992\right)$.

La cuantificación del contenido de flavonoides se realizó según el procedimiento De Stalikas ${ }^{10}$, empleando tricloruro de aluminio. Se preparó un extracto etanólico de ajo en polvo de 50 $\mathrm{mg} / \mathrm{mL}$ a $80^{\circ} \mathrm{C}$. Agitándose vigorosamente, se dejó por 2 horas y luego se centrifugó a 4000 rpm por 10 minutos. La reacción en el tubo fue: $360 \mu \mathrm{L}$ del extracto, $40 \mu \mathrm{L}$ de $\mathrm{AlCl}_{3} 5 \%$ y 600 $\mu \mathrm{L}$ de agua bidestilada. Después de 30 minutos se leyó a $425 \mathrm{~nm}$. Se preparó una curva de calibración empleando como referencia quercetina (Q), con concentraciones de 10 a 100 $\mu \mathrm{g} / \mathrm{mL} .\left(\mathrm{y}=0,0687 \mathrm{x} ; \mathrm{R}^{2}=0,9996\right)$. El resultado es expresado como equivalentes de quercetina (EQ) en $\mathrm{mg} / \mathrm{g}$ masa seca.

\section{Evaluación de metales asociados a defensa antioxidante}

Se determinó $\mathrm{Cu}, \mathrm{Zn}, \mathrm{Mn}$, Fe y Se mediante el análisis multilelemental de la muestra por ICPMS (Inductively coupled plasma-mass spectrometry) siguiendo el protocolo EPA 3050B para la preparación de la muestra y el protocolo EPA 6020A, para el procedimiento analítico. En resumen, se trabajó con $0,3417 \mathrm{~g}$ de muestra seca, se procedió a una digestión con adición repetida de ácido nítrico y peróxido de hidrógeno. La digestión resultante se llevó a un volumen de $50 \mathrm{~mL}$. Se usó agua desionizada. Los resultados se reportan en $\mathrm{mg} / \mathrm{kg}$.

\section{Evaluación de la actividad antioxidante in vitro}

Se realizó dos ensayos:

a) Inducción de Estrés Oxidativo en un modelo in vitro en eritrocitos humanos

Se trabajó con la metodología de Murphy et $\mathrm{al}^{11}$. Todos los ensayos se realizaron por triplicado de manera independiente.

Se obtuvo los glóbulos rojos (GR) $6 \mathrm{~mL}$ de sangre venosa con heparina en ayuno. La muestra de extracto acuoso de ajo se preparó en tres concentraciones: $100 \mathrm{mg} / \mathrm{mL}, 10 \mathrm{mg} / \mathrm{mL}$ y $1 \mathrm{mg} / \mathrm{mL}$.

Se preparó 8 tubos denominados de la siguiente manera:

T1: referencia, GR y PBS, T2: estrés, GR y peróxido de hidrógeno, T3: estrés y extracto de allium $10 \mathrm{mg} / \mathrm{mL}$, T4: estrés y extracto de allium $1 \mathrm{mg} / \mathrm{mL}, \mathbf{T 5}$ : estrés y extracto de allium 0,1 $\mathrm{mg} / \mathrm{mL}$, T6: referencia y extracto de allium $10 \mathrm{mg} / \mathrm{mL}$, T7: referencia y extracto de allium 1 $\mathrm{mg} / \mathrm{mL}$, T8: referencia y extracto de allium $0,1 \mathrm{mg} / \mathrm{mL}$.

Para los ensayos, se preparó GR a una concentración de $25 \%$ en buffer fosfato salino (PBS) y $\mathrm{NaN}_{3} 2 \mathrm{mM}$. Se colocó el tubo en baño de maría a $37^{\circ} \mathrm{C}$ con agitación por 30 minutos.

Se preparó la batería de los 8 tubos, cada uno con un volumen total de $0,8 \mathrm{~mL}$. Se colocó en cada tubo $0,32 \mathrm{~mL}$ de GR y $80 \mu \mathrm{L}$ de ajo según la denominación de los tubos indicados arriba, se completa con PBS. Se continuó con el baño de maría a $37^{\circ} \mathrm{C}$ por 30 minutos y luego se añadió $0,4 \mathrm{~mL}$ de peróxido de hidrógeno a los tubos T2, T3, T4 y T5; nuevamente baño de maría $37^{\circ} \mathrm{C}$ por 60 minutos. Después de concluido el tratamiento, se procedió a medir TBARS.

\section{Determinación de especies reactivas al ácido tiobarbitúrico (TBARS)}

Se midió, por espectrofotometría, el complejo MDA-TBA formado por la reacción del malondialdehído (MDA) con el ácido tiobarbitúrico (TBA) a la longitud de $535 \mathrm{~nm}$.

Procedimiento: En tubos con tapa rosca se colocó $0,3 \mathrm{~mL}$ de muestra y $0,6 \mathrm{~mL}$ de ácido tricloroacético al 20\%; se llevó a baño de maría hirviente por 15 minutos. Se retiró y se añadió $0,9 \mathrm{~mL}$ de ácido tiobarbitúrico $0,67 \%$ preparado en $\mathrm{HCl} 0,25 \mathrm{~N}$. Se continuó con baño en las mismas condiciones durante 30 minutos, luego se enfrió y se llevó a centrifugación 6000 rpm durante 10 minutos. Los resultados se obtienen empleando el coeficiente de extinción del complejo MDA-TBA, $1,56 \times 10^{5} \mathrm{M}^{-1} \mathrm{~cm}^{-1}$. 
b) Segundo ensayo: Los carbonilos proteicos se producen por el ataque de radicales libres sobre las estructuras proteicas; la presencia de este grupo funcional es evidencia del daño oxidativo a proteínas. El daño oxidativo se realizó empleando peróxido de hidrógeno y $\mathrm{Cu}^{+2}$. La detección de carbonilos se realizó con el 2,4-dinitrofenilhidracina (DNPH) ${ }^{12}$.

Para producir el daño oxidativo, en cada tubo se colocó $0,5 \mathrm{~mL}$ de albúmina $10 \mathrm{mg} / \mathrm{mL}$ y/o ajo $10 \mathrm{mg} / \mathrm{mL}$; se añadió $0,1 \mathrm{~mL}$ de sulfato cúprico $5 \mathrm{mM}$ y $0,1 \mathrm{~mL}$ de peróxido de hidrógeno 10 $\mathrm{mM}$ y completó con agua bidestilada hasta un volumen total de $5 \mathrm{~mL}$. Las muestras no oxidadas se prepararon en el mismo volumen empleando las mismas cantidades exceptuando la adición de los reactivos oxidantes.

Se llevó a agitación a temperatura ambiente durante media hora. Luego se trabajó con dos tubos para cada sistema, preparados colocando $1 \mathrm{~mL}$ de muestra y $4,0 \mathrm{~mL}$ de $\mathrm{HCl}$ 0,25 M para los tubos control; y el mismo volumen de DNFH $10 \mathrm{mM}$ para los tubos de reacción.

Se colocó en baño a temperatura ambiente con agitación durante 1 hora. Se añadió 5,0 $\mathrm{mL}$ de TCA 20\%; se dejó por 10 minutos en baño de hielo. Se centrifugó a $7000 \mathrm{rpm}$ por cinco minutos. El sedimento se lavó con 4,0 mL de TCA 10\% y luego, por tres veces, con 4,0 mL de una mezcla de etanol: acetato de etilo (1:1). El precipitado se disolvió con 2,0 mL de guanidina $6 \mathrm{M}$, durante 10 minutos a $37^{\circ} \mathrm{C}$, agitando.

Se llevó a leer a $365 \mathrm{~nm}$. Para los cálculos se empleó el $\varepsilon=2,2 \times 10^{4} \mathrm{M}^{-1} \mathrm{~cm}^{-1}$.

Los resultados se expresan en $\mathrm{nmol} / \mathrm{mg}$ proteína.

Determinación de proteínas: La muestra control disuelta en guanidina se leyó a $280 \mathrm{~nm}$. Se preparó una curva de calibración con una solución de albúmina $2 \mathrm{mg} / \mathrm{mL}$ disuelto en la solución de guanidina con concentraciones de $0,25,0,5,1,0$ y $1,5 \mathrm{mg} / \mathrm{mL}$. La lectura de proteínas de cada tubo se determinó en el tubo control después de añadirle la guanidina. La curva de calibración dio la recta: $\mathrm{y}=0,4791 \mathrm{x}+0,0253 ; \mathrm{R}^{2}=0,9984$.

Todos los ensayos se hicieron por duplicado en tres determinaciones independientes.

\section{RESULTADOS Y DISCUSIÓN}

El contenido de alicina, metabolito secundario mejor conocido del Allium sativum resultó ser $1,31 \mathrm{mg} / \mathrm{mL}$ de extracto, expresado en ajo fresco pelado corresponde a 2,8 $\mathrm{mg}$ alicina/g. Este resultado se encuentra dentro de lo reportado por la literatura: $2,5 \mathrm{a} \mathrm{4,5} \mathrm{mg} / \mathrm{g}$ de peso fresco. Resultados reportados por Eagling y Sterling ${ }^{13}$, sobre ajo australiano, muestran valores de alicina entre 7 y $9 \mathrm{mg} / \mathrm{g}$ de ajo fresco, determinados por HPLC, mencionan que son los de mayor contenido a nivel mundial pues otros estudios dan resultados desde $1,7 \mathrm{mg} / \mathrm{g}$ de peso fresco. Estos autores hacen notar que los mayores niveles de alicina se producen en regiones con climas cálidos, secos y con suelos de alto $\mathrm{pH}$, debido a que en estas condiciones se favorece la captación de sulfato, además de las condiciones de almacenamiento.

La cuantificación de tioles de bajo peso molecular resultó en $0,95 \pm 0,118 \mu \mathrm{mol} \mathrm{Cis/g} \mathrm{de} \mathrm{masa}$ seca en presencia del reductor ácido ascórbico; en ausencia de este agente no se pudo detectar; lo que significa que los tioles se encuentran oxidados.

Sobre los metabolitos oxigenados los resultados para los polifenoles y flavonoides fue 2,52+ $0,01 \mathrm{mg}$ EAG/g masa seca y $1,71 \pm 0,00 \mathrm{mg}$ EQ/g masa seca, respectivamente. El contenido de polifenoles también fue ensayado en medio metanólico (1,45 mg EAG/ g masa seca); el extracto acuoso fue significativamente superior $(\mathrm{p}<0,001)$ al extracto metanólico; lo interesante de este resultado es que la literatura consigna de manera preferente la extracción metanólica para la determinación de polifenoles, significa que en el caso del ajo investigado estos metabolitos secundarios son principalmente hidrosolubles, constituyendo una gran ventaja por la economía de este solvente y su aplicación en preparados nutricionales y otros. 
Comparando los resultados de los extractos acuoso y metanólico expresados en mg EAG/g masa fresca resultaron superiores a otros ajos reportados en la literatura. Sin embargo, Gorinstein et $\mathrm{al}^{5}$ reportaron que el contenido de polifenoles en ajos de Polonia, un valor de 19,4 $\mathrm{mg}$ EAG/g masa seca.

En la determinación de flavonoides, también se empleó dos solventes alcohólicos: metanol y etanol. Los resultados fueron significativamente mayores con el solvente etanol $(p<0,001)$. Resalta para ambas determinaciones polifenoles y flavonoides, la menor extracción con metanol; importante, dada la toxicidad de este solvente.

Comparando con otras hortalizas, Pérez-Gregorio et $\mathrm{al}^{14}$ mostraron que el contenido de flavonoides en cebolla fresca, fue de $390 \mathrm{mg} \mathrm{EQ} / \mathrm{kg}$ masa fresca y este contenido aumentó a alrededor de $570 \mathrm{mg}$ EQ/kg de masa fresca, cuando fue expuesto a la luz durante 16 días. El bulbo de ajo huaralino exhibió un contenido de $647 \mathrm{mg} \mathrm{EQ} / \mathrm{kg}$ de masa fresca.

Los resultados de la evaluación de los metales asociados al sistema antioxidante se observa en la tabla 1.

Tabla 1. Minerales determinados por Análisis Multielemental mediante Inducción de Plasma Acoplada.

\begin{tabular}{lc|cc}
\hline Analito & $\begin{array}{c}\text { Concentración } \\
\mathrm{mg} / \mathrm{kg}\end{array}$ & Analito & $\begin{array}{c}\text { Concentración } \\
\mathrm{mg} / \mathrm{kg}\end{array}$ \\
\hline Cobre & 2,8 & $\mathrm{Mn}$ & $<0,1$ \\
Hierro & 16,7 & Cinc & 31,2 \\
Potasio & 11230 & Selenio & $<0,03$ \\
\hline
\end{tabular}

La tabla 1 muestra la detección de estos minerales en el ajo como masa seca; los metales antes mencionados fueron detectados aunque estos valores son ligeramente menores a los reportados por USDA National Nutrient Database for Standard Reference: Fe 56,5; Cu 5,33; Mn 9,79; Se 0,239 mg/kg, excepto para el cinc que fue 31,2 mg/kg, ligeramente mayor a 29,9 $\mathrm{mg} / \mathrm{kg}$. Es importante mencionar que el mineral más abundante es el potasio. Estos resultados pueden diferir por la variedad del bulbo, clima, suelo y condiciones agronómicas que resultan en diversos contenidos de minerales.

Los ensayos que se realizaron para determinar las actividades antioxidantes in vitro se muestran en las figuras 1 y 2 .

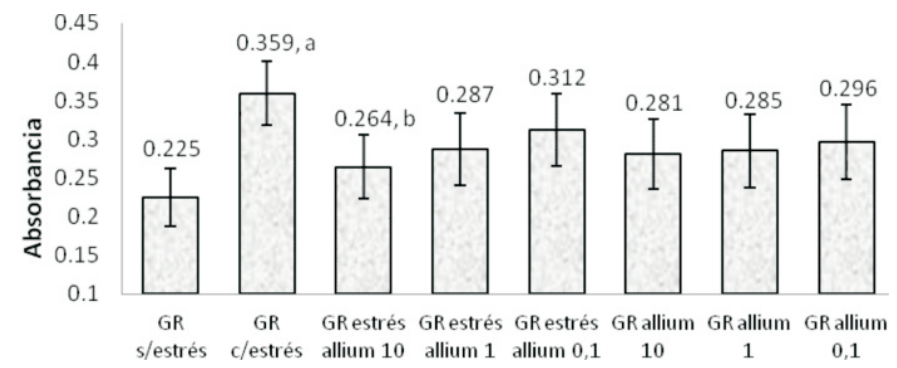

Figura 1. Prevención de la formación de TBARS por efecto de la presencia del extracto acuoso de Allium sativum variedad huaralino en un modelo de estrés oxidativo in vitro en eritrocito humano. 
a: $p<0,05$ frente al grupo de glóbulos rojos sin estrés (GRs/estrés), $b: p<0,05$ frente al grupo de glóbulos rojos con estrés (GRclestrés).

Los extractos acuosos de ajo huaralino preparados a tres concentraciones también exhibieron significativo efecto protector in vitro sobre la lipoperoxidación (TBARS) de los glóbulos rojos humanos (figura 1) a la concentración de $10 \mathrm{mg} / \mathrm{mL}(\mathrm{p}<0,05)$. Los tratamientos con 1 y 0,1 $\mathrm{mg} / \mathrm{mL}$ presentaron una disminución de $20 \%$ y $13 \%$ con respecto al grupo glóbulo rojo oxidado pero sin significancia estadística.

En la misma figura se observa que la presencia del extracto de ajo en el medio de los glóbulos rojos también produce un ligero incremento, no significativo, en comparación con la muestra control; este ligero incremento fue casi homogéneo en las tres concentraciones de extracto; es decir, independiente de la concentración.

En la literatura se reporta que el extracto de ajo, en especial sus componentes orgánicos disulfuros, puede atravesar la membrana del eritrocito y realizar diversas actividades en el medio citoplasmático, probablemente modificando diversas proteínas. Estas actividades ejercen modificaciones favorables. Takasu et $\mathrm{al}^{3}$ han reportado los

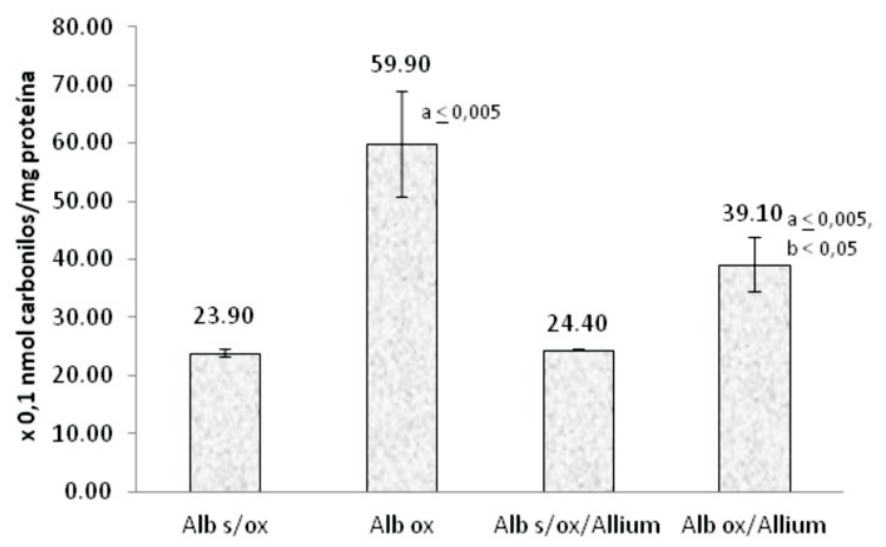

Figura 2. Prevención de la formación de carbonilos proteicos por efecto de la presencia del extracto acuoso de Allium sativum variedad huaralino.

$a: p \leq 0,005$ frente a albúmina sin oxidación (Alb s/ox), $b: p<0,05$ frente a albúmina oxidada (Alb $o x)$

beneficios del consumo de ajos envejecidos en pacientes con anemia falciforme en un tratamiento por un mes, produjeron disminución significativa $(\mathrm{p}<0,05)$ de $58,9 \%$ inicial a $29,8 \%$ al final del tratamiento.

Ameen et $\mathrm{al}^{15}$ también han reportado los efectos benéficos en un modelo experimental con ratas hembras sometidas a la toxicidad pulmonar de crisotilo (silicato) por periodos de hasta 180 días. Los principales resultados fueron una inhibición en la formación de TBARS y de la actividad de la enzima arilhidrocarburo hidroxilasa hacia los 90 y 180 días, así como la recuperación del GSH. También se observó inducción de la actividad de la glutatión Stransferasa; entendiéndose con ello que el extracto de ajo puede dar protección mediante cambios en el sistema enzimático de destoxificación. 
Los resultados que ser reportan en el presente trabajo dan cuenta de ese efecto protector medido por la disminución de la formación de TBARS.

En el caso de la detección de carbonilos proteicos puede observarse en la figura 2 la efectividad del cobre y el peróxido de hidrógeno en la oxidación de la albúmina $(\mathrm{p}<0,005)$ y el efecto protector en la formación de los carbonilos del ajo huaralino en comparación con la muestra de albúmina oxidada $(\mathrm{p}<0,05)$. El contenido de carbonilos en los ensayos de los tubos de ajo y en el ajo oxidado fue los más bajos, 0,14 y 0,23 nmol carbonilos/mg proteína, respectivamente. Se interpreta que el extracto de ajo no contribuye al incremento de carbonilos, aun cuando este ha sido sometido a un daño oxidativo. Lo expuesto permite plantear el beneficio del extracto de ajo en la protección al daño oxidativo a proteínas. La formación de carbonilos producto del daño oxidativo a proteínas, ha sido demostrado en sistemas biológicos in vitro e in vivo, por lo que la búsqueda de metabolitos naturales que puedan proteger o remover estos carbonilos será importante con fines fitoterapéuticos. Estudios realizados por Ashraf et $\mathrm{al}^{6}$ dan pruebas que los extractos vegetales son capaces de atenuar la formación de carbonilos, de manera semejante al mostrado en este trabajo.

\section{CONCLUSIONES}

El extracto acuoso de Allium sativum variedad huaralino contiene metabolitos secundarios azufrados (alicina y otros) y oxigenados (polifenoles y flavonoides), así como metales asociados al sistema antioxidante que explicarían el efecto de protección antioxidante in vitro disminuyendo la formación de carbonilos proteicos y de peroxidación lipídica.

\section{AGRADECIMIENTOS}

Los autores agradecen a la Facultad de Medicina de la UNMSM por el apoyo parcial económico y a la Estación Experimental Donoso de la ciudad de Huaral por la donación de las muestras.

\section{REFERENCIAS}

1. Banerjeea SK, Maulika M, Mancahandab SC, Dindac AK, Guptaa SK and Maulik SK. Dose-dependent induction of endogenous antioxidants in rat heart by chronic administration of garlic. Life Sciences. 2002; 70:1509-1518.

2. Takasu J, Uykimpang R, Sunga MA, Amagase H and Niihara Y. Aged garlic extract is a potential therapy for sickle-cell anemia. J. Nutr. 2006; 136: 803S-805S.

3. Pendbhaje NS, Narang AP, Pathan SM, Raotole SA and Pattewar SV. Ethnopharmacoloy, pharmacognosy and phytochemical profile of allium sativum L.: A Review. Pharmacologyonline. 2011;2:845-853.

4. Ceci R, Reyes M, Duranti G, Dimauro Y, Quaranta F, Pittaluga M, Sabatini S, Caserotti P, Parisi P, Parisi A and Caporossi D. Oxidative stress responses to a graded maximal exercise test in older adults following explosive-type resistance training. Redox Biology. 2014; 2:65-72.

5. Gorinstein, S; Leontowicz, H; Leontowicz, M; Namiesnik, J; Najman, K; Drzewiecki, J; Cvikrová, M; Martincová, O; Katrich, E and Trakhtenberg, S. Comparison of the main bioactive compounds and antioxidant activities in garlic and white and red onions after treatment protocols. J. Agric. Food Chem. 2008; 56: 4418-4426.

6. Ashraf M, Butt MS, Pasha I and Sheikh MA. Phytochemical profiling of pakistani garlic varieties; special attention to antioxidant status. Pakistan Journal of Science. 2013; 65 (4): $478-485$. 
7. Miron T, Shin I, Feigenblat G, Weiner L, Mirelman D, Wilchek M and Rabinkov A. A spectrophotometric assay for allicin, alliin, and alliinase (alliin lyase) with a chromogenic thiol: reaction of 4-mercaptopyridine with thiosulfinates. Analytical Biochemistry. 2002; 307: 76-83.

8. Di Simplicio P, Cacace MG, Lusini L, Giannerini F, Giustarini D and Rossi R. Role of protein -sh groups in redox homeostasis - the erythrocyte as a model system. Archives of Biochemistry and Biophysics. 1998; 355(2): 145-152.

9. Anesini C, Ferraro GE and Filip R. Total polyphenol content and antioxidant capacity of commercially available tea (camellia sinensis) in Argentina. J. Agric. Food Chem. 2008; 56: 9225-9229.

10. Stalikas CD. J. Extraction, separation, and detection methods for phenolic acids and flavonoids. J. Sep. Sci. 2007; 30: 3268-3295.

11. Murphy PG, Davies MJ, Columb MO and Stratford N. Effect of propofol and thiopentone on free radical mediated oxidative stress of the erythrocyte. British Journal of Anaesthesia. 1996; 76: 536-543.

12. Punchard NA and Kelly FJ, editors. Free radicals a practical approach. London: Oxford University Press; 1996.

13. Eagling D and Sterling S. A cholesterol-lowering extract from garlic. Australia: Rural Industries Research and Development Corporation. 2000; 18pp.

14. Pérez-Gregorio M.R., García-Falcón M.S., Simal-Gándara J. Flavonoids changes in fresh-cut onions during storage in different packaging systems. Food Chemistry. 2011; 124: 652-658.

15. Ameen M, Musthapa MS, Abidi P, Ahmad I and Rahman Q. Garlic attenuates chrysotilemediated pulmonary toxicity in rats by altering the phase I and phase II drug metabolizing enzyme system. Journal of Biochemical and Molecular Toxicology. 2003; 17 (6): 366-371. DOI: 10.1002/jbt.10100. 